\title{
Point-of-Care Ultrasound Can Suggest COVID-19
}

\author{
Ossama Maadarani, Zouheir Bitar, Tamer Zaalouk, Mohammad Mohsen, Ragab Elshabasy \\ Internal Medicine Department, Ahmadi Hospital - Kuwait Oil Company, Kuwait
}

Doi: 10.12890/2020_001915 - European Journal of Case Reports in Internal Medicine - ㅇ EFIM 2020

Received: $24 / 07 / 2020$

Accepted: 30/07/2020

Published: $25 / 08 / 2020$

How to cite this article: Maadarani O, Bitar Z, Zaalouk T, Mohsen M Elshabasy R. Point-of-care ultrasound can suggest COVID-19. EJCRIM 2020;7: doi:10.12890/2020_001915.

Conflicts of Interests: The Authors declare that there are no competing interests.

This article is licensed under a Commons Attribution Non-Commercial 4.0 License

\section{ABSTRACT}

Coronavirus disease 2019 (COVID-19) is caused by severe acute respiratory syndrome coronavirus 2 (SARS-CoV-2) and the World Health Organization (WHO) declared it a pandemic on 11 March 2020. Point-of-care ultrasound (POCUS) is a real-time bedside tool used by physicians to guide rapid, focused and accurate evaluation in order to identify or rule out various pathologies. We describe the case of an elderly man who had fallen at home 3 days previously and was hypoxic at presentation to the emergency department (ED). POCUS in the ED helped to identify a combination of lung and vascular involvement that indicated COVID-19 infection, which was confirmed by a laboratory test.

\section{LEARNING POINTS}

- COVID-19 is a contagious disease caused by SARS-CoV-2 that attacks endothelial cells and most organs, resulting in different manifestations and clinical scenarios.

- Point-of-care ultrasound in the emergency room including lung ultrasound (LUS) and focused echocardiography (FECHO) can be useful in identifying pulmonary and vascular manifestations of COVID-19 disease during the current pandemic.

- Characteristic LUS signs suggesting bilateral interstitial pneumonia in addition to signs of acute right ventricular strain suggesting pulmonary embolism on FECHO raised the suspicion of COVID-19 infection in our patient.

\section{KEYWORDS}

COVID-19, POCUS, B lines, flashing phenomenon, pulmonary embolism

\section{INTRODUCTION}

Point-of-care ultrasound (POCUS) is a rapid, reliable imaging technique performed at the patient's bedside for diagnostic and therapeutic purposes and is used mainly by critical care and emergency department physicians. The ability to look inside the human body in real time, especially in critically ill patients, without the need for transfer to the radiology department allows rapid and accurate investigation. Realtime, reverse transcriptase-polymerase chain reaction (rRT-PCR) assay to detect SARS-CoV-2 nucleic acid is the current test of choice to diagnose COVID-19 infection ${ }^{[1]}$. COVID-19 is a contagious disease caused by SARS-CoV-2 that attacks endothelial cells and most organs, resulting in different manifestations and clinical scenarios. Our patient presented with vague symptoms and was afebrile and hypoxic. Rapid POCUS assessment revealed a combination of findings suggestive of interstitial pneumonia and acute right ventricular strain, which during current pandemic raised the possibility of COVID-19 infection that was confirmed by rRT-PCR.

\section{CASE DESCRIPTION}

An 82-year-old male patient who had injured himself in a fall at home presented to the emergency department complaining of facial pain with a superficial wound to his nose. He denied loss of consciousness, headache, nausea, vomiting or chest pain. He is independent, ambulatory and has a known history of diabetes mellitus, coronary artery disease and coronary artery bypass graft surgery many years previously. 
His medications include aspirin, bisoprolol $1.25 \mathrm{mg}$ once daily, and insulin glargine. He had never smoked and denied alcohol consumption. He was afebrile, his blood pressure (BP) was 120/80 mmHg, heart rate (HR) was 80 beats/minute, and $\mathrm{O}_{2}$ saturation (SatO ${ }_{2}$ ) was $97 \%$ on room air. Neurological examination showed no deficits or lateralization signs, while other examinations were unremarkable. Chest radiography (Fig. 1) showed increased bronchovascular markings bilaterally which were more prominent on the right lung base, similar to previous radiographs. There were no rib fractures. Computed tomography of the head and brain showed evidence of a small fractured nasal bone and no evidence of recent events in the brain. The patient was seen by a surgeon and ENT clinician and was discharged home.

After 3 days he again presented to the ED complaining of mild dizziness, right shoulder pain and mild chest discomfort. He remained afebrile but looked ill and dehydrated. His BP was $90 / 60 \mathrm{mmHg}$, HR was 105 beats/minute, SatO ${ }_{2}$ was $88 \%$ on room air and respiratory rate was 26 breaths per minute. He denied cough, shortness of breath, abdominal pain or diarrhoea. He denied contact with anyone who was sick. Arterial blood gas analysis in ambient air confirmed type 1 respiratory failure $\left(\mathrm{PaO}_{2} 9.4 \mathrm{kPa}\right)$. His ECG showed sinus tachycardia with nonspecific changes, while chest radiography (Fig. 2) revealed some infiltrates mainly on the right lung and which seemed new compared to the image taken 3 days previously.

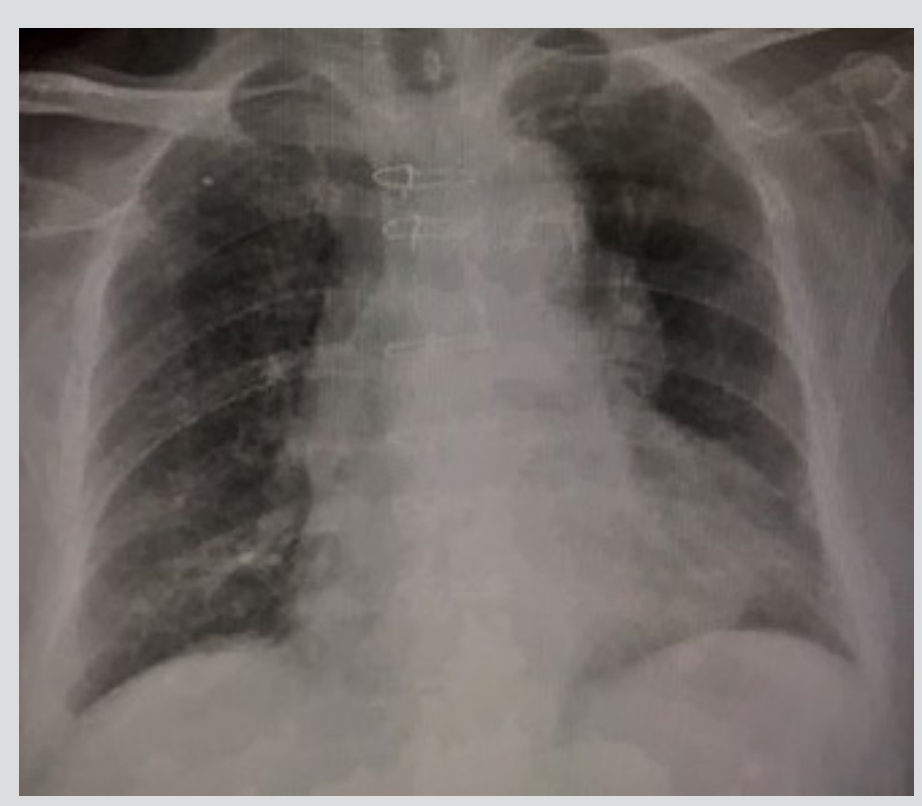

Figure 1. Chest radiograph on the first visit to the emergency department

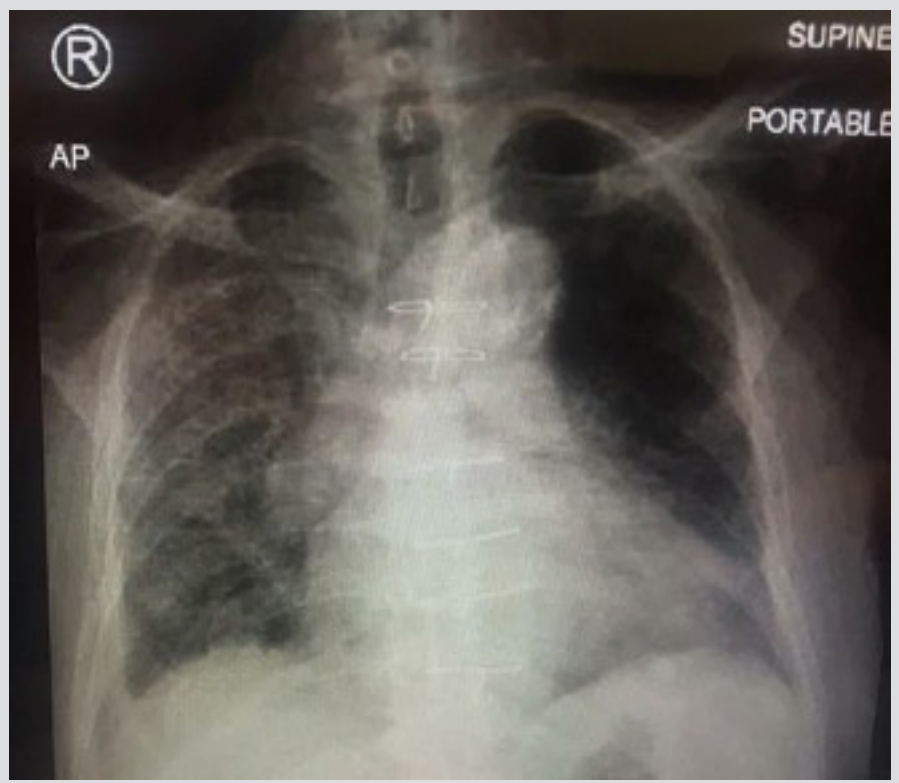

Figure 2. Chest radiograph on the second visit to the emergency department

During POCUS assessment, lung ultrasound (LUS) scanning of six areas on each side using a curvilinear probe revealed in the right posterior and lateral lower areas patchy thickened and unsmooth pleural lines with dense confluent $B$ lines (white lung area) with absence of $A$ lines in the affected areas (Fig. 3) and spared areas where the pleural line looks smooth with A lines. In the left posterior lower area, a thickened and fragmented pleural line with subpleural consolidations and a dense B line starting from consolidations appeared intermittently and moving with the pleural sliding giving the appearance of 'flashing phenomena' (Fig. 4). The scan of right and left upper anterior areas showed scattered B lines with intermittent smooth and unsmooth pleural lines. The LUS findings suggested patchy involvement of both lungs with a pattern similar to interstitial pneumonia with the right lung more affected.

Focused echocardiography (FECHO) showed a dilated right ventricle (RV) with a ratio of $>1: 1$, and severe hypokinesia of the RV. The interventricular septum (IVS) was shifted towards the left ventricle (LV) causing the classic D-shape of the LV (Fig. 5) and mild to moderate tricuspid regurgitation with mild to moderate pulmonary hypertension. Comparison with echocardiography findings from a few months previously showed these changes were new and suggested an acute right ventricular strain probably related to pulmonary embolism (PE). Such LUS and FECHO findings during the current pandemic raised the suspicion of COVID-19 infection as a cause of the patient's clinical presentation. Laboratory findings were significant for mild leucocytosis with lymphopenia, raised D-dimer, troponin I and pro-BNP, and evidence of acute kidney injury. The patient was started on intravenous fluids and high-flow $\mathrm{O}_{2}$, a nasopharyngeal swab for SARS-CoV-2 was taken and he was moved to the critical care unit. He remained hypotensive and hypoxic. Thrombolytic therapy according to the PE protocol was administered and the patient was intubated and mechanically ventilated. The nasopharyngeal swab for SARS-CoV-2 returned positive. Unfortunately, the patient remained hypoxic and died after a few hours. 


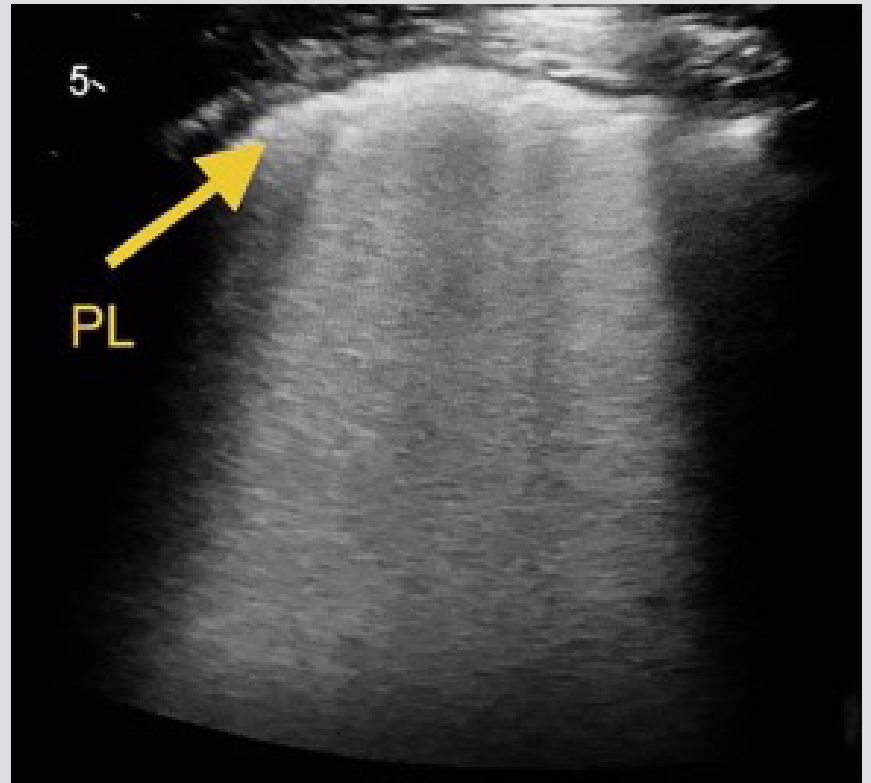

Figure 3. Right posterior and lower area: thickened pleural line (PL) and confluent B lines (white lung)

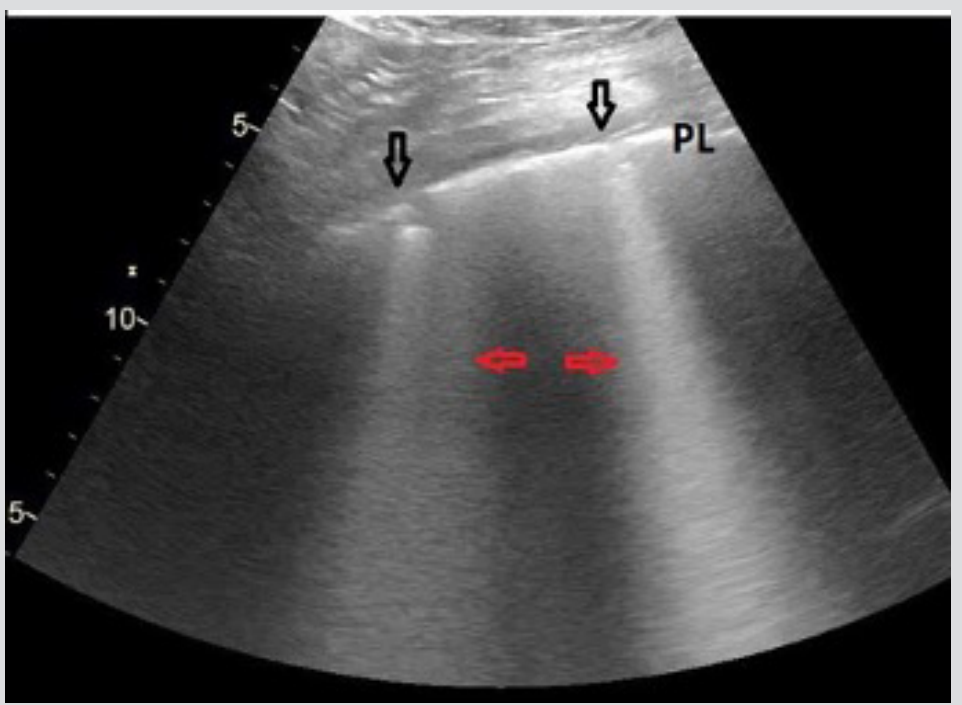

Figure 4. Flashing phenomenon: left posterior and lower area - subpleural consolidations (black arrows) and dense B lines (red arrows) appearing on and off and moving with pleural sliding. PL, pleural line

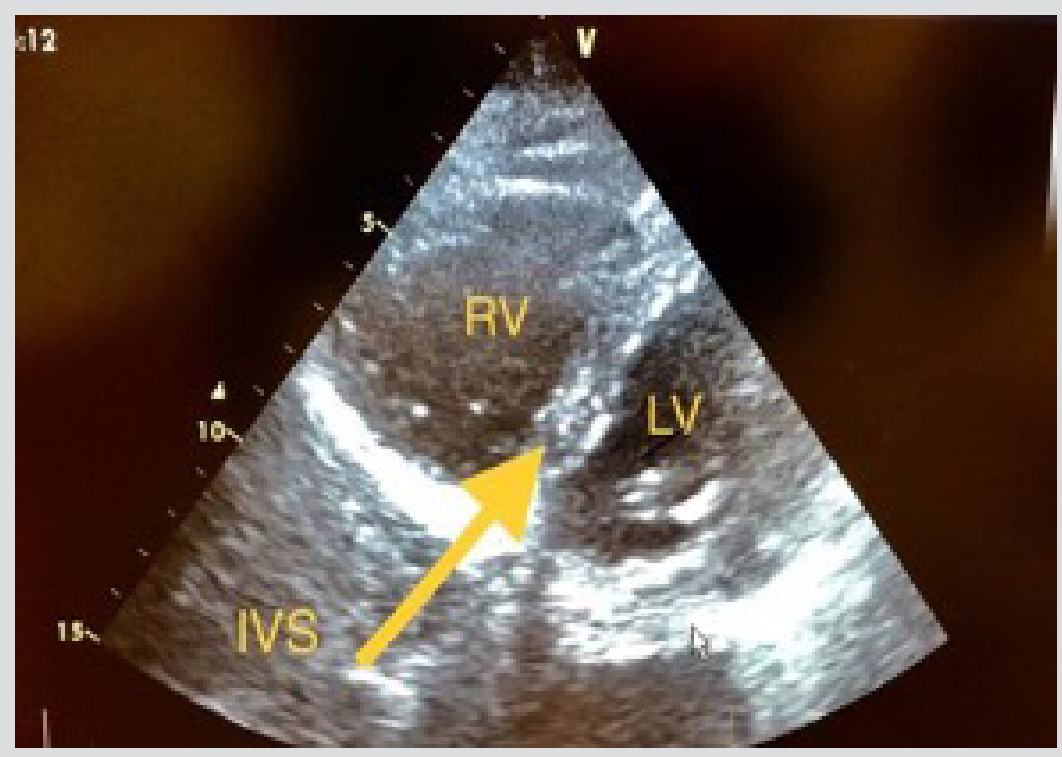

Figure 5. Left parasternal short axis view. RV dilated with $R V: L V$ ratio of more than 1:1 and the D-shaped LV is suggestive of right heart strain IVS, interventricular septum; LV, left ventricle; $R V$, right ventricle

\section{DISCUSSION}

POCUS is used by physicians at the bedside for rapid, focused and accurate evaluation to identify or rule out various pathologies. The pulmonary and cardiovascular systems are important targets for POCUS where LUS and FECHO can provide great detail to identify changes in the patient's condition in real time ${ }^{[2]}$. Several protocols for POCUS are currently available and used in different clinical scenarios such as undifferentiated dyspnoea, hypoxia or shock, and include the bedside lung ultrasound in emergency (BLUE), the rapid assessment of dyspnoea with ultrasound (RADiUS) and the rapid ultrasound in shock (RUSH) protocols.

SARS-CoV-2 is the virus responsible for the current COVID-19 pandemic ${ }^{[3]}$ and has tropism to the lower respiratory tract ${ }^{[1]}$ with striking manifestations in the pulmonary system as demonstrated by CT of the chest which shows characteristic bilateral interstitial pneumonia with patchy and asymmetric lesions distributed mainly in the periphery of the lung ${ }^{[4]}$. As most pulmonary lesions in COVID-19 patients are distributed mainly in the peripheral areas of both lungs and since LUS relies on the artifacts of peripulmonary lesions to suggest the diagnosis, this makes LUS a precise tool to detect these lesions. 
The correlation between LUS and CT chest finding was demonstrated by Jin et al. who concluded that LUS was an effective tool and superior to standard chest radiography for assessing the severity of pulmonary disease in COVID-19 [5].

Different studies have reported that the following LUS findings are commonly found in COVID-19 patients: subpleural consolidations mainly in the posterior and inferior areas of the lung, separate and confluent B lines, thickened or irregular pleural lines, and signs of air bronchograms. Identifying such findings during a pandemic helps the physician to detect patients who are likely infected; however, the above ultrasound findings may not be specific to COVID-19 as they are seen in other viral pneumonias ${ }^{[5]}$.

Volpicelli et al. described an artifact observed in most patients with COVID-19 pneumonia and called it 'light beam', which is seen when a broad band-shaped, vertical artifact moves rapidly with sliding, at times creating an 'on-off' effect as it appears and disappears from the screen ${ }^{[6]}$. In our case we observed a similar finding when a dense vertical artifact starting from subpleural consolidation appeared on and off creating a flashing phenomenon.

The effect of SARS-CoV-2 on endothelial cells plays an important role in vascular injury that contributes to pulmonary, cardiovascular and other manifestations. Endothelial dysfunction and endotheliitis are considered the basis of thrombus formation and result in COVID-19associated thromboembolic insult of different organs and can partially explain the hypercoagulable state commonly associated with patients infected with SARS-CoV-2 ${ }^{[7]}$. Thromboembolic manifestations of COVID-19 have been described in several reports. In the ED and critical care units, POCUS in the appropriate clinical context is an effective tool to rapidly diagnose acute pulmonary embolism associated with right heart strain and possible thrombus in transit, and guide further treatment ${ }^{[8]}$. Ultrasound signs of right heart strain include bowing of the IVS into the LV, right ventricular dilatation and systolic dysfunction including McConnell's sign, possible tricuspid regurgitation, a dilated inferior vena cava and visible right heart thrombus.

In our case, the LUS findings of bilateral patchy lesions in the posterior and inferior areas with intermittent spared areas were consistent with a picture of interstitial pneumonia. These results, together with the FECHO findings of acute right heart strain and PE in the current pandemic raised the possibility of COVID-19 infection which was confirmed with rRT-PCR.

\section{CONCLUSION}

POCUS is an important bedside tool that can help identify pulmonary and vascular manifestations of COVID-19 disease during the current pandemic.

\section{REFERENCES}

1. Phelan AL, Katz R, Gostin LO. The novel coronavirus originating in Wuhan, China: challenges for global health governance. JAMA 2020 Jan 30. doi: 10.1001/jama.2020.1097 [Epub ahead of print].

2. Volpicelli G, Elbarbary M, Blaivas M, Lichtenstein DA, Mathis G, Kirkpatrick AW, et al. International evidence-based recommendations for point-of-care lung ultrasound. Intensive Care Med 2012;38(4):577-591.

3. Zhu N, Zhang D, Wang W, Li X, Yang B, Song J, et al.; China Novel Coronavirus Investigating and Research Team. A novel coronavirus from patients with pneumonia in China, 2019. N Engl J Med 2020;382:727-773.

4. Wu J, Wu X, Zeng W, Guo D, Fang Z, Chen L, et al. Chest CT findings in patients with coronavirus disease 2019 and its relationship with clinical features. Invest Radiol 2020;55(5):257-261.

5. Jin YH, Cai L, Cheng ZS, Cheng H, Deng T, Fan YP, et al. A rapid advice guideline for the diagnosis and treatment of 2019 novel coronavirus (2019-nCoV) infected pneumonia (standard version). Mil Med Res 2020;7:4.

6. Volpicelli G, Lamorte A, Villén T. What's new in lung ultrasound during the COVID-19 pandemic. Intensive Care Med 2020;46:1445-1448.

7. Ciceri F, Beretta L, Scandroglio AM, Colombo S, Landoni G, Ruggeri A, et al. Microvascular COVID-19 lung vessels obstructive thromboinflammatory syndrome (MicroCLOTS): an atypical acute respiratory distress syndrome working hypothesis. Crit Care Resusc 2020;22(2):95-97.

8. Kahl N, Gabriel C, Lahham S, Thompson M, Hoonpongsimanont W. Point-of-care ultrasound diagnosis of pulmonary embolism with thrombus in transit. Clin Pract Cases Emerg Med 2019;3(1):11-12. 